\title{
Characterization of a Novel Allergen, a Major IgE-Binding Protein from Aspergillus flavus, as an Alkaline Serine Protease
}

\author{
Chia-J ung Yu,* Shyh-Horng Chiou,† Wei-Yu Lai,* Bor-Luen Chiang,‡ and Lu-Ping Chow*

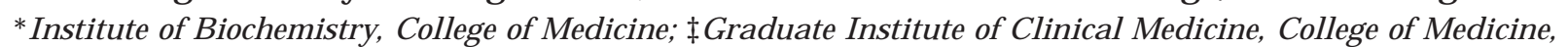 \\ National Taiwan University; and †Institute of Biological Chemistry, Academia, Taipei, Taiwan
}

\begin{abstract}
Aspergillus species of fungi have been known to be one of the most prevalent aeroallergens. One important A. flavus allergen (Asp fl 1) was identified by means of immunoblotting with a serum pool of allergic patients on a two-dimensional electrophoretic gel. The CDNA coding for Asp fl 1 was cloned and sequenced. The clone encodes a full-length protein of $\mathbf{4 0 3}$ amino acid precursors of $42 \mathrm{kDa}$. After cleavage of a putative signal peptide of 21 amino acids and a prepeptide of 100 amino acids, a mature protein of 282 amino acids was obtained with a molecular mass of $33 \mathrm{kDa}$ and a pl of 6.3. A degree of identity was found in a range of 27 to $84 \%$ among related allergens derived from bacteria allergen subtilisin, mold allergen Pen c 1, and virulence factor of A. fumigatus. Recombinant Asp fl 1 (rAsp fl 1) was cloned into vector pQE-30 and expressed in E. coli M15 as a histidine-tag fusion protein and purified to homogeneity. The IgE binding capacity of rAsp fl 1 was tested by immunoblotting using a serum pool of Aspergillus-allergic patients. Recombinant allergen cross-reacted strongly with IgE specific for natural Asp fl 1 and Pen c 1, indicating that common IgE epitopes may exist between allergens of $A$. flavus and P. citrinum. $\odot 1999$ Academic Press

Key Words: mold allergens; IgE-binding activity; alkaline serine protease; Aspergillus flavus.
\end{abstract}

The role of fungi in producing respiratory allergy and asthma has long been established. Many fungi are the major source of allergens, such as Cladosporium, Alternaria, Fusarium, Penicillium and Aspergillus species (1). Aspergillus sp. is abundant indoors and is thought to be an important inducer of allergic asthma and allergic rhinitis. Of the genus Aspergillus, A. flavus was found ubiquitously in temperate and tropical

Abbreviations: RACE, rapid amplification of CDNA ends; TBS, Tris-buffered saline; IPTG, isopropyl-1-thio- $\beta$-D-galactopyranoside; ABPA, allergic bronchopulmonary aspergillosis. areas of the world. It accounts for a variety of respiratory disorders, such as hypersensitivity pneumonitis, I gE-mediated asthma and allergic bronchopulmonary aspergillosis (ABPA) (2), but its role in antigenic/ allergenic activity is unclear.

A number of allergenic components from Aspergillus sp. are associated with pulmonary diseases in which the diagnosis can be confirmed by the demonstration of specific IgE antibodies in patients' sera. The major 18-kDa antigen (Asp $f$ ) has been purified and characterized as a member of the mitogillin family of cytotoxins (3), however mRNA was only detected in A. fumigatus but not in other Aspergillus species (4). A $65-k D a$ I gE-binding protein shows homology with heat shock protein 90 family (5). Another 37 kDa ConAnonbinding allergen of $A$. fumigatus was shown to react significantly with IgE antibody in patients with ABPA, but does not elicit reaction in Aspergillussensitive subjects with asthma (6). Furthermore, the catalase subunit (7) and superoxide dismutase (8) allergens have been purified to homogeneity from water soluble extracts of A. fumigatus. Several enzymes from Aspergillus sp. are common aeroallergens such as pectinase I, cellulase, flaviastase and glucoamylase from A. niger, plus $\alpha$-amylase and lactase from A. oryzae (9).

It was reported from our laboratory that an allergen isolated from $P$. citrinum belongs to the proteinase family of alkaline serine proteases. This major allergen, referred to as Pen c 1 , has been purified, cloned and expressed (10). To explore the possibility whether some similar serine protease may also be a significant allergen of Aspergillus species, we describe herein the identification and molecular cloning of a novel serine protease from the mold A. flavus. The cloned allergen was designated Asp fl 1 according to the recommendation of I nternational Union of I mmunological Societies (11). Furthermore, Asp fl 1-encoding cDNA was subcloned into the expression plasmid pQE 30 and expressed in E. coli. The recombinant protein was puri- 
fied and analyzed on immunoblots in an effort to characterize its immunological reactivities with patients' IgE and crossreactivity with natural Asp fl 1 and another mold related allergen Pen c 1.

\section{MATERIALS AND METHODS}

Patients' sera and monoclonal antibody. For the identification of IgE-reactive proteins, we used a mixture of sera from four Aspergillus-sensitive patients (Pharmacia CAP System, CAP denoted as score 4 according to manufacturer's manual; Pharmacia Diagnostics AB, U ppsala, Sweden). Sera from non-allergic individuals were collected and used as a negative control. Allergen was verified by immunoblotting using our mAb 55A antibodies against the Pen C 1 of P. citrinum, prepared previously (10).

Allergen extracts. A. flavus used in the preparation of allergenic extracts was obtained from the Food Industry Research \& Development Institute, Hsinchu, Taiwan (CCRC 32242). Fungal matts of A. flavus containing both spores and mycelia were collected from CzapekDox broth medium (Difco, Detroit, MI, USA).

Protein extraction and two-dimensional PAGE. About one gram of lyophilized matts was ground in a mortar with liquid nitrogen, ground mycelia and spores were then mixed with phosphate-buffered saline extraction buffer at $4^{\circ} \mathrm{C}$ for $1 \mathrm{~h}$ with constant stirring. After centrifugation, the supernatant was precipitated with trichloroacetic acid to a final concentration of $5 \%$. The resultant precipitate was stored at $-70^{\circ} \mathrm{C}$. Dried powder $(2 \mathrm{mg})$ was suspended in $100 \mu \mathrm{l}$ of loading buffer (9.0 M urea, 2\% carrier ampholytes, $60 \mathrm{mM}$ DTT, $0.5 \%$ Triton-X 100 and $0.003 \%$ bromophenol blue) and 2-dimensional PAGE was carried out by a procedure previously described (12). In the first dimension IEF, an immobilized pH gradient of 3 to 10 was used on a horizontal electrophoresis system, Multiphor II (Pharmacia Co., Sweden); for the second dimension, SDS-PAGE was performed.

SDS-PAGE and immunoblotting. SDS-PAGE was essentially carried out as described by Laemmli (13) using a separation gel of $12.5 \%$ polyacrylamide. For immunoblotting detection of Asp fl 1 , the gel was subjected to electroblotting to a PVDF membrane after SDS-PAGE followed by immunological analysis using diluted patients' pooled sera at $4^{\circ} \mathrm{C}$ for $16 \mathrm{~h}$. After washing, the membrane blots were incubated with alkaline phosphatase-conjugated monoclonal anti-human IgE antibodies (Pharmingen, San Diego, CA, USA) at room temperature for $1 \mathrm{~h}$. The binding patterns were visualized by a substrate solution consisting of NBT/BCIP (10).

$\mathrm{N}$-terminal microsequencing. The blotted proteins were visualized with Coomassie Brilliant Blue R-250 stain. After destaining, protein spots of interest were excised and amino acid sequencing was performed on a PROCISE 494 protein sequencer (Perkin EImer/ABI, Foster City, CA, USA) equipped with an on-line detection of phenylthiohydantoin derivatives. The partial sequences were compared for homologies to known proteins by using the Basic Local Alignment Search Tool (BLAST) algorithm.

Preparation of mRNA from A. flavus, PCR amplification, and cloning. One gram of the mycelia was ground in a mortar filled with liquid nitrogen. After grinding RNA isolation was performed according to the manufacturer's protocol of Life Sciences (Petersburg, FL, USA). Poly(A) ${ }^{+}$RNA of A. flavus was purified using the QuickPrep mRNA preparation kit (Pharmacia, Uppsala, Sweden). Doublestrand CDNA was synthesized from mRNA using the Marathon cDNA amplification kit (Clontech Laboratories, Palo Alto, CA, USA) according to the manual. After $\mathrm{N}$-terminal sequencing, the following specific primers were designed based on the partial sequence of the conserved $A$. oryzae alkaline serine protease (14) for $5^{\prime}$ primer $\left(5^{\prime}\right.$ ATGCAGTCCATCAAGCGTACCTTG-3') and ' $3^{\prime}$ primer (5'-TTAAGCGTTACCGTTGTAGGCAAG-3' with the stop codon at the end of
3' coding region) deduced from the $C$-terminal amino acid sequence. Coding sequence of the Asp fl 1 gene was thus amplified by PCR. The amplified products were analyzed by electrophoresis and subcloned into pGEM-T (Promega, Madison, WI, USA). The sequence of the amplified product was confirmed using the AmpliTaq Cycle Sequencing Kit (Perkin EImer/ABI, Foster City, CA, USA).

Expression and purification of recombinant Asp fl 1 ( $r$ Asp fl 1). Expression of recombinant Asp fl 1 protein in E. coli was carried out as described (15). The full-length sequence encoding mature Asp fl 1 was obtained from A. flavus CDNA by PCR amplification. Primers were designed to include a Bam $\mathrm{HI}$ site at the $5^{\prime}$ end and a HindlII site at the $3^{\prime}$ end of the CDNA. The primers used were 5'-GCGGATCCGGCCTGACTACCCAGAAG-3' (sense primer) and 5'-CCCAAGCTTTTAAGCGTTACCGTTGTAGGC-3' (antisense primer). Purified PCR products were ligated into a Bam $\mathrm{HI}$ $\mathrm{H}$ indlII restriction sites of the $\mathrm{PQE}-30$ expression vector containing the sequence encoding the $6 \times \mathrm{H}$ is affinity tag (QIAGEN, Chatsworth, CA, USA). The ligation mixture was then subcloned into $E$. coli M15 strain cells (QIAGEN). For induction of gene expression, isopropyl- $\beta$-D-thiogalactopyranoside (IPTG) was added in a final concentration of $1 \mathrm{mM}$ once the culture reached $A_{600}=$ 0.6 . The cells were harvested at 2 -h intervals. The correct reading frame was re-checked by sequencing, and the recombinant protein was purified by affinity chromatography in a column of $\mathrm{Ni}^{2+}$-charged resin (QIAGEN). Recombinant $\mathrm{His}_{6}$-Asp fl 1 protein was eluted from the resin with $8 \mathrm{M}$ urea, $20 \mathrm{mM}$ Tris- $\mathrm{HCl} / \mathrm{pH}$ 7.9, containing $60 \mathrm{mM}$ imidazole/0.5 M NaCl as described (10). Immunological detection was performed with serum pool from Aspergillus sensitized patients.

Inhibition of IgE reactivity to native Asp fl 1 and Pen c 1 . Asp fl 1 was isolated from supernatants of 3-day culture, growing in $0.3 \%$ soybean meal with vigorous shaking at $30^{\circ} \mathrm{C}$. The medium was filtered through cheesecloth, ammonium sulfate was then added to the supernatant in a concentration of $80 \%$ saturation. The resultant precipitate was collected by centrifugation, re-dissolved in $10 \mathrm{mM}$ sodium phosphate buffer, $\mathrm{pH} 7.8$, and dialyzed against the same buffer. After dialysis, the clear supernatant was applied to a DE-52 cellulose column which was pre-equilibrated with the buffer. The flow-through fractions were collected and applied to a CM-52 cellulose column pre-equilibrated with $10 \mathrm{mM}$ sodium acetate buffer, $\mathrm{pH}$ 5.0. The column was eluted with a linear gradient of $0-0.04 \mathrm{M}$ sodium chloride in the same buffer. IgE-binding activity of the fractions was assessed by immunoblotting analysis with a pool of patients' sera allergic to Aspergillus. For immunoblot inhibition studies, $1 \mathrm{ml}$ sample of sera was preincubated with purified allergen $\mathrm{rAsp}$ fl $1, B S A$ and buffer as control for $2 \mathrm{~h}$ at $4^{\circ} \mathrm{C}$. The preadsorbed sera was then tested for their I gE reactivities against native Asp fl 1 and Pen c 1 (16).

Sequence comparison and construction of a phylogenetic tree A software package of LaserGene for the Apple Macintosh computer from DNASTAR, Inc. was used for the estimation of sequence homology based on percent similarity and divergence among different cDNA sequences of mold allergens and other serine proteases. Percent divergence is calculated by comparing sequence pairs in relation to the phylogenetic tree. On the other hand the percent similarity is estimated by comparing sequences directly without accounting for phylogenetic relationships. Phylogenetic tree was then constructed using the algorithm of Hein (17) in the MegAlign programs of the package. It is a multiple-sequence alignment program that builds trees as it aligns DNA or protein sequences using a combination of distance matrix and approximate parsimony methods. This method constructs multiple-alignment by imposing restrictions based on evolutionary relatedness of the aligned sequences, which is useful to align highly evolved gene families that have clear evolutionary relationships. 
A

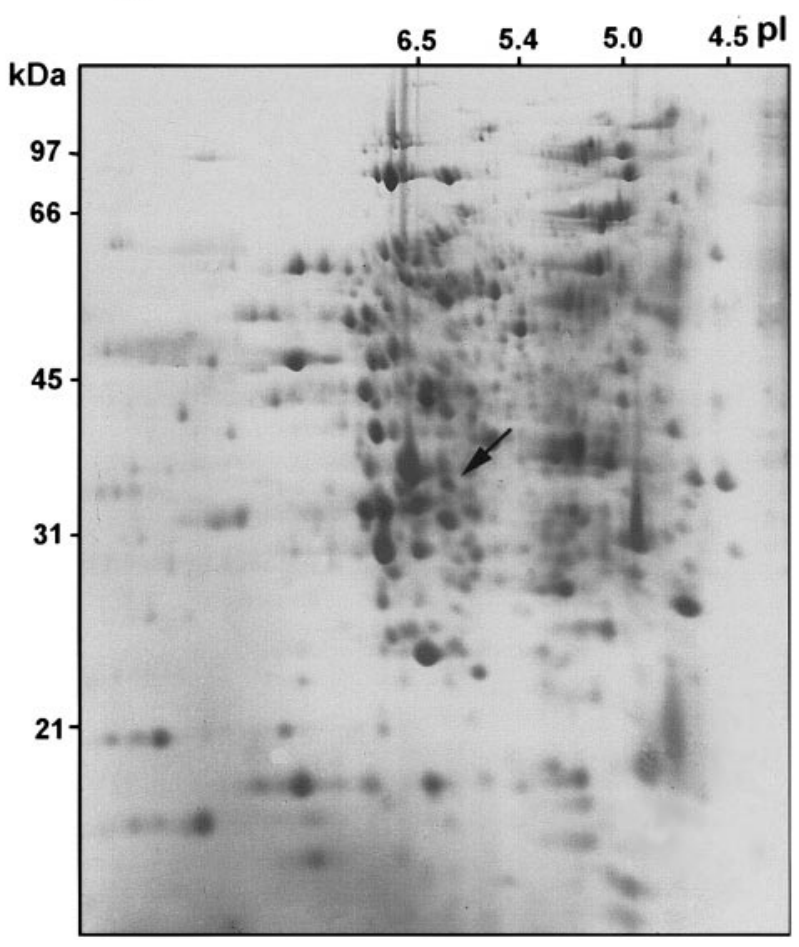

B

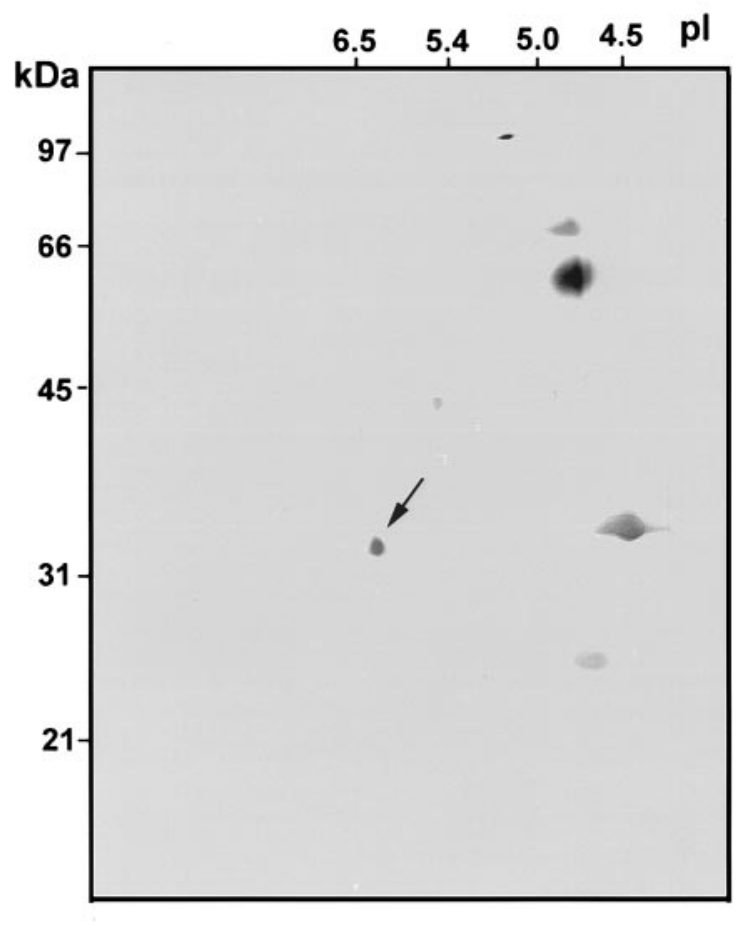

FIG. 1. Two-dimensional immunoblot analysis. Procedures for $2 D-P A G E$ are as described in the Materials and Methods section. (A) P. citrinum crude extract was visualized by staining the blots with Coomassie brilliant blue. (B) 2D-PAGE of crude extract in (A) was immunoblotted with IgE antibodies of the atopic human serum pool. The arrow indicates the immunoreactive spot to IgE antibodies, which corresponds to the allergen of serine protease family.

\section{RESULTS AND DISCUSSION}

Allergen Identification, 2D-I mmunoblotting, and $\mathrm{N}$-Terminal Sequencing

In order to characterize antigens recognized by allergen-sensitive sera, two-dimensional immunoblotting was carried out with the crude extract of A. flavus. Figure 1A shows the Coomassie blue-stained protein profile of the crude protein extract. About 200 distinct protein spots were detected on the $2 \mathrm{D}$ gel. To identify spots corresponding to the allergens, I gE-binding spots in 2D-PAGE were visualized by immunoblotting using sera from Aspergillus-sensitized patients. After immunoblotting, seven different spots with molecular masses of 28 to $65 \mathrm{kDa}$ and pl values ranging from about 5.0 to greater than 7.0 were shown to be reactive to IgE antibodies (Fig. 1B). To identify the IgE-reactive serine protease in $A$. flavus, seven immunoreactive protein spots were characterized again with monoclonal antibody mAb 55A directed specifically against alkaline serine proteinase, a well-described mold allergen termed Pen c 1 from P. citrinum (data not shown). F or negative control, no positive spots were detected when the negative serum was used. The arrow in Fig. $1 \mathrm{~A}$ indicates the protein spot corresponding with that showing positive reaction in immunoblotting of Fig.
1B. N-terminal sequence of this $33 \mathrm{kDa}$ protein with a pl of 6.3 was determined to be GLTTQKSAPWG. When compared with the Genebank, it was found that the sequence exhibited high sequence identity to alkaline serine protease of A. oryzae (14).

\section{Sequence Analysis of the cDNA and Derived Amino Acid Sequence of the Asp fl 1 Gene}

The nucleotide sequence of the Asp fl 1 gene is shown in Fig. 2. The Asp fl 1 cDNA is an open-reading frame of 1212 nucleotides, coding for 403 amino acids. This reading frame starts with a ATG codon and ends with a stop codon at nucleotide position 1,212. The encoded protein has a predicted molecular mass of $42 \mathrm{kDa}$. At the amino terminal region, a series of uncharged amino acid residues with a high content of hydrophobic amino acids were found. Based on the signal sequence cleavage prediction (18), the most likely cleavage site in the present protein would be between Ala-20 and Pro- 21 . The signal sequence that is expected to direct transport of the nascent polypeptide chain across the ER membrane into the ER lumen (19). The N-terminal sequence of the Asp fl 1, GLTT, matched with the deduced a.a. sequence of the gene at a.a. 122-125 within the open reading frame. This indicates that Asp fl 1 has 
$\begin{array}{llllllllllllllllllllllllll}1 & \mathbf{M} & \mathbf{Q} & \mathbf{S} & \mathbf{I} & \mathbf{K} & \mathbf{R} & \mathbf{T} & \mathbf{L} & \mathbf{L} & \mathbf{L} & \mathbf{L} & \mathbf{G} & \mathbf{A} & \mathbf{I} & \mathbf{L} & \mathbf{P} & \mathbf{A} & \mathbf{V} & \mathbf{L} & \mathbf{G} & \mathbf{A} & \mathbf{P} & \mathbf{V} & \mathbf{Q}\end{array}$ ATG CAG TCC ATC AAG CGT ACC TTG CTC CTC CTC GGA GCT ATC CTT CCC GCG GTC CTC GGT GCC CCT GTG CAG

$\begin{array}{lllllllllllllllllllllllll}25 & \mathbf{E} & \mathbf{T} & \mathbf{R} & \mathbf{R} & \mathbf{A} & \mathbf{A} & \mathbf{E} & \mathbf{K} & \mathbf{L} & \mathbf{P} & \mathbf{G} & \mathbf{K} & \mathbf{Y} & \mathbf{I} & \mathbf{V} & \mathbf{T} & \mathbf{F} & \mathbf{K} & \mathbf{P} & \mathbf{G} & \mathbf{I} & \mathbf{D} & \mathbf{E} & \mathbf{A}\end{array}$ GAA ACC CGC CGG GCC GCT GAg AAG CTT CCT GGA AAG TAC ATT GTC ACA TTC AAg CCC GeC ATT GAC GAG GCA

$\begin{array}{llllllllllllllllllllllllll}49 & \mathbf{K} & \mathbf{I} & \mathbf{Q} & \mathbf{E} & \mathbf{H} & \mathbf{T} & \mathbf{T} & \mathbf{W} & \mathbf{A} & \mathbf{T} & \mathbf{N} & \mathbf{I} & \mathbf{H} & \mathbf{Q} & \mathbf{R} & \mathbf{S} & \mathbf{L} & \mathbf{E} & \mathbf{R} & \mathbf{R} & \mathbf{G} & \mathbf{A} & \mathbf{T} & \mathbf{G}\end{array}$

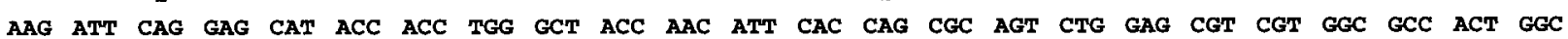

$\begin{array}{llllllllllllllllllllllllllll}73 & G & D & \text { L } & \text { P } & \text { V } & \text { G } & \text { I } & \text { E } & \text { R } & \text { N } & \text { Y } & \text { K } & \text { I } & \text { N } & \text { K } & \text { F } & \text { A } & \text { A } & \text { Y } & \text { A } & \text { G } & \text { S } & F & \text { D }\end{array}$ GGT GAT CTT CCT GTC GGT ATT GAG CGC AAC TAC AAG ATC AAC AAG TTC GCC GCC TAT GCA GGC TCT TTC GAC

$\begin{array}{llllllllllllllllllllllllll}97 & \mathbf{D} & \mathbf{A} & \mathbf{T} & \mathbf{I} & \mathbf{E} & \mathbf{E} & \mathbf{I} & \mathbf{R} & \mathbf{K} & \mathbf{N} & \mathbf{E} & \mathbf{D} & \mathbf{V} & \mathbf{A} & \mathbf{Y} & \mathbf{V} & \mathbf{E} & \mathbf{E} & \mathbf{D} & \mathbf{Q} & \mathbf{I} & \mathbf{Y} & \mathbf{Y} & \mathbf{I}\end{array}$ GAT GCT ACC ATT GAG GAG ATT CGC AAG AAC GA GAT GTT GCC TAC GTC GAG GAG GAC CAg ATC TAC TAC CTC

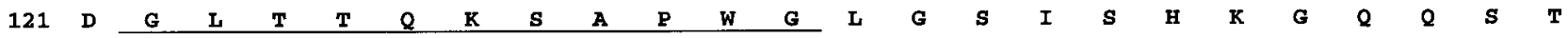
GAT GGC CTG ACT ACC CAG AAg AGT GCC CCC TGG GGT CTG GGC AGC ATT TCC CAC Aag GGC CAg CAG AGC ACC

$\begin{array}{llllllllllllllllllllllllll}145 & D & Y & I & Y & D & \text { T } & \text { S } & \text { A } & \text { G } & \text { E } & \text { G } & \text { T } & \text { Y } & \text { A } & \text { Y } & \text { V } & \text { V } & \text { D } & \text { S } & \text { G } & \text { V } & \text { N } & \text { V } & \text { D }\end{array}$

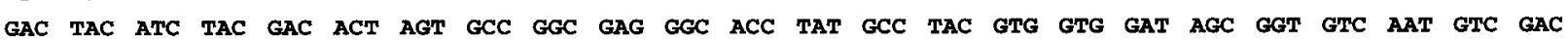

$\begin{array}{llllllllllllllllllllllllll}169 & \text { H } & \text { E } & \text { E } & \text { F } & \text { E } & \text { G } & \text { R } & \text { A } & \text { S } & \text { K } & \text { A } & \text { Y } & \text { N } & \text { A } & \text { A } & \text { G } & \text { G } & \text { Q } & \text { H } & \text { V } & \text { D } & \text { S } & \text { I } & \text { G }\end{array}$

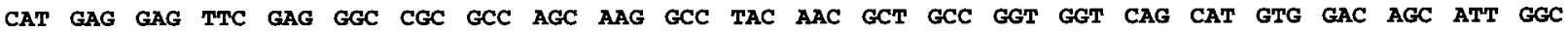
*

$\begin{array}{llllllllllllllllllllllllllll} & \text { H } & \text { G } & \text { T } & \text { H } & \text { V } & \text { S } & \text { G } & \text { T } & \text { I } & \text { A } & \text { G } & \text { K } & \text { T } & \text { Y } & \text { G } & \text { I } & \text { A } & \text { K } & \text { K } & \text { A } & \text { S } & \text { I } & \text { L } & \text { S }\end{array}$ CAT GGC ACC CAC GTT TCC GGC ACC ATT GCT GGC AAG ACT TAT GGT ATC GCC AAg Aag GCC AGC ATC CTT TCG

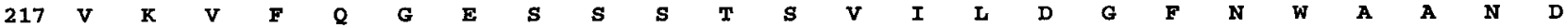
GTC AAA GTT TTC CAG GGT GAA TCG AGC AGC ACT TCC GTC ATT CTT GAC GGC TTC AAC TGG GCT GCC AAC GAC

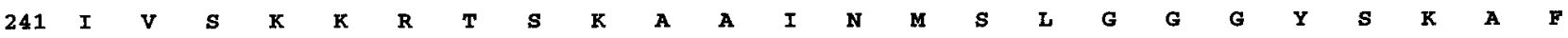
ATT GTT AGC AAG AAG CGT ACC AGC AAg GCT GCA ATC AAC ATG AGC TTG GGC GGT GGC TAC TCT AAG GCT TTC

$\begin{array}{lllllllllllllllllllllllll}265 & \mathbf{N} & \mathbf{D} & \mathbf{A} & \mathbf{V} & \mathbf{E} & \mathbf{N} & \mathbf{A} & \mathbf{F} & \mathbf{E} & \mathbf{Q} & \mathbf{G} & \mathbf{V} & \mathrm{L} & \mathbf{S} & \mathbf{V} & \mathbf{V} & \mathbf{A} & \mathbf{A} & \mathbf{G} & \mathbf{N} & \mathbf{E} & \mathbf{N} & \mathbf{S} & \mathbf{D}\end{array}$ AAC GAT GCG GTC GAG AAC GCA TTC GAG CAG GGT GTT CTC TCG GTT GTC GCT GCC GGT AaC GAG AAC TCT GAT

$\begin{array}{llllllllllllllllllllllllll}289 & \mathbf{A} & \mathbf{G} & \mathbf{Q} & \mathbf{T} & \mathbf{S} & \mathbf{P} & \mathbf{A} & \mathbf{S} & \mathbf{A} & \mathbf{P} & \mathbf{D} & \mathbf{A} & \mathbf{I} & \mathbf{T} & \mathbf{V} & \mathbf{A} & \mathbf{A} & \mathbf{I} & \mathbf{Q} & \mathbf{K} & \mathbf{S} & \mathbf{N} & \mathbf{N} & \mathbf{R}\end{array}$ GCC GGC CAA ACC AGC CCT GCC TCT GCC CCT GAT GCC ATC ACT GTT GCC GCT ATC CAG Aag AGC AAC AAC CGC

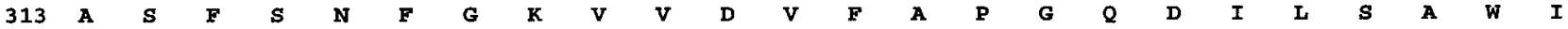
GCC AGT TTC TCC AAC TTT GGC AAG GTC GTT GAC GTC TTC GCT CCC GGT CAA GAT ATC CTT TCT GCC TGG ATT

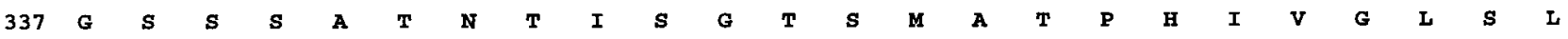
GGC TCT TCC TCT GCC ACC AAC ACC ATC TCT GGT ACC TCC ATG GCT ACT CCC CAC ATT GTC GGC CTG TCC CTT

$\begin{array}{llllllllllllllllllllllllll} & 361 & \mathbf{Y} & \text { L } & \text { A } & \text { A } & \text { I } & \text { E } & \text { N } & \text { L } & \text { D } & \text { G } & \text { P } & \text { A } & \text { A } & \text { V } & \text { T } & \text { K } & \text { R } & \text { I } & \text { E } & \text { E } & \text { L } & \text { A } & \text { T } & \text { K }\end{array}$

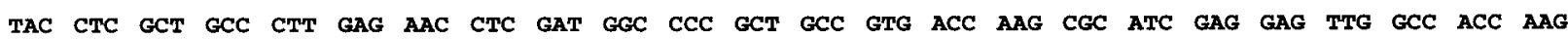

1152

$\begin{array}{llllllllllllllllllll}385 & D & V & V & K & D & \text { V } & \text { K } & G & S & \text { P } & \text { N } & \text { L } & \text { L } & \text { A } & \text { Y } & \text { N } & \text { G } & \text { N } & \text { A }\end{array}$

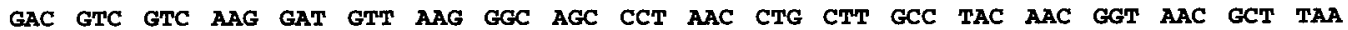

FIG. 2. Nucleotide and deduced amino acid sequences of an Asp fl 1 clone. The nucleotide sequence is shown below the amino acid sequence. The numbers on the right of the figure indicate the positions of the nucleotide sequence. The numbers on the left of the figure indicate the positions of the deduced amino acid sequence. $\mathrm{N}$-terminal segment determined by protein sequencing is underlined. The catalytic important Asp, His and Ser residues are marked by asterisks. Nucleotide sequence has been submitted to GeneBank/E MBL/DDJ B Databases under the accession number AF 137272.

a rather long propeptide consisting of 392 amino acids. This propeptide contains a 100 amino-acid segment that shows $100 \%$ identify with a corresponding segment in the propeptide of the A. oryzaeserine protease.
Most proteases of the serine protease family possess a precursor structure containing a long propeptide (20). The function of the propeptide is thought to keep the protease from autolysis until it is processed (21). 

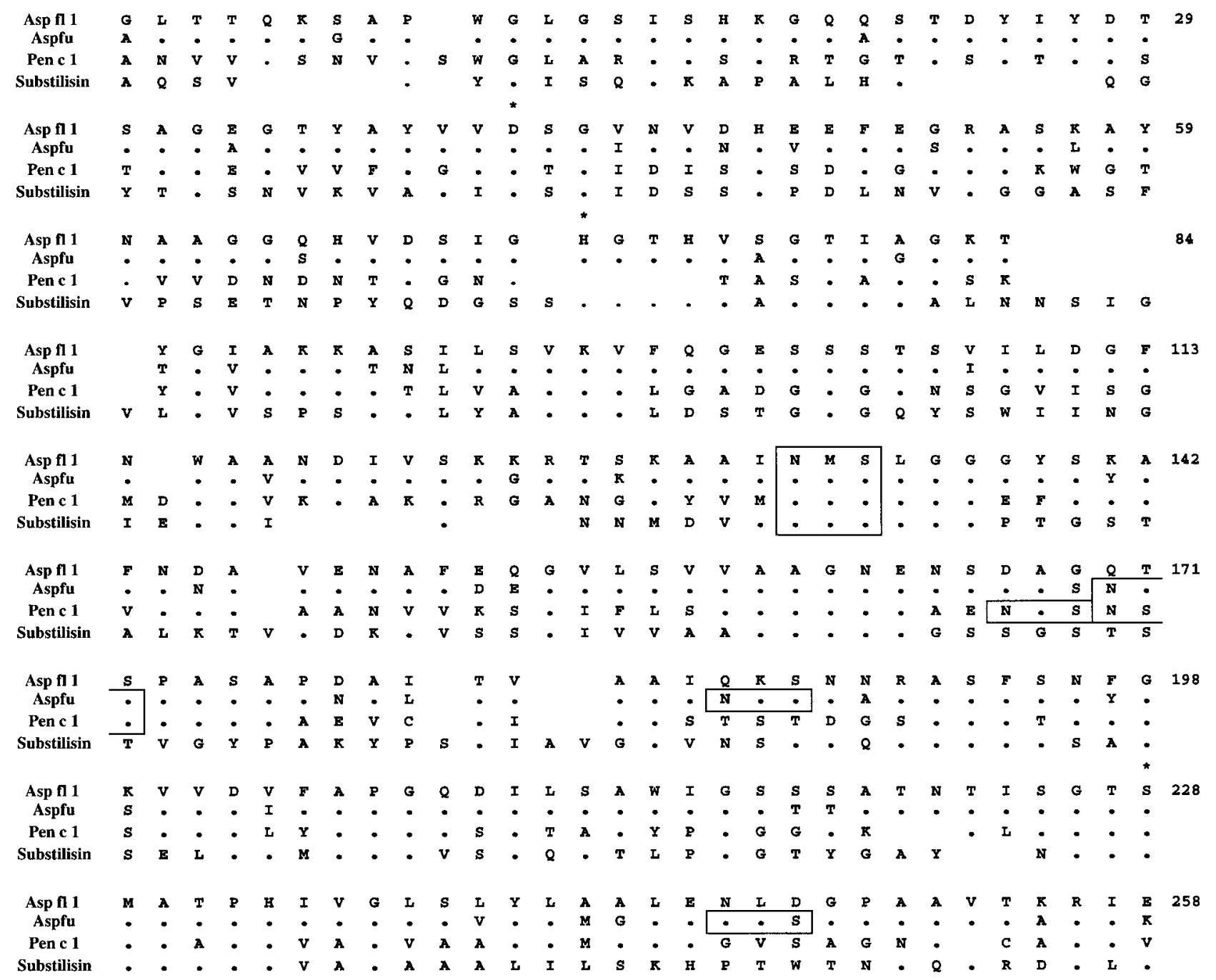

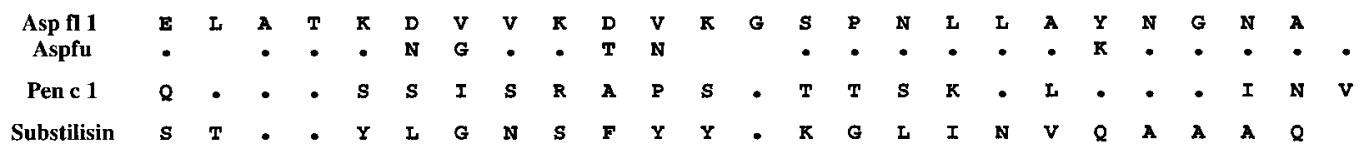

FIG. 3. Multiple sequence alignment of Asp $f l$ and other related allergens. The numbering system is based on the sequence of Asp fl 1 as reference. The gaps are introduced for optimal alignment and maximum homology among all compared sequences. I dentical amino acids are indicated by dots. The highly conserved and consensus amino acid residues involved in the active site are indicated by asterisks. The potential consensus sequences for $\mathrm{N}$-glycosylation are boxed.

\section{Comparison of Asp fl 1 Protein Sequences with Other Related Allergens}

We compared the amino acid sequence of our allergen with other serine protease allergens. The protein sequence showed varied extents of sequence similarity with known related allergens and virulence factor (Fig. 3). It shows $83.9 \%, 40.0 \%$ and $27.3 \%$ identity to elastase of A. fumigatus (22), Pen c 1 (10) and subtilisin (23), respectively. Residues of the catalytic triad of Asp fl 1 (Asp 41, His 72, and Ser 228) are immediately recognized by homology comparison and suggested to play a crucial role for catalytic activity in serine pro- tease (24). There is only one potential glycosylation site at Asn ${ }^{132} \mathrm{MetSer}$, whereas four such regions are found in $\mathrm{A}$. fumigatus elastase and three such regions in $\mathrm{P}$. citrinum Pen c 1 . It is noteworthy that Asp fl 1 contains no cysteine residues. High-degree identity was also found with that of elastolytic protease of Aspergillus fumigatus, which has been described as a virulence factor in invasive aspergillosis (25). It was suggested that mold elastase can induce human epithelial cell detachment in allergic patients and might play a crucial role in triggering the allergic response (26). Asp fl 1 showed $40 \%$ sequence identity with Pen c 1 , implying 
A

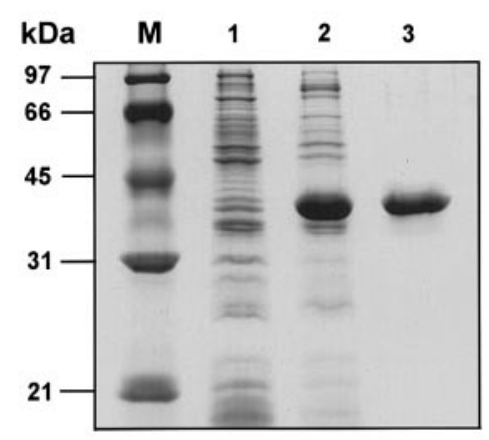

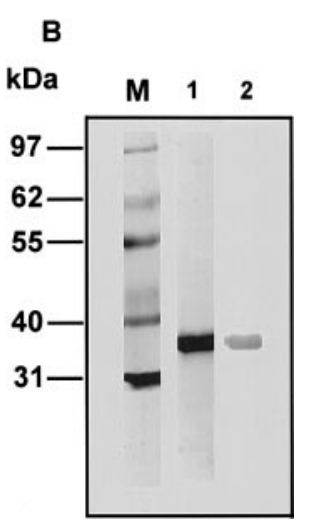

FIG. 4. I mmunological characterization of recombinant Asp fl 1 ( $\mathrm{rAsp} f \mathrm{fl}$ ). (A) SDS-PAGE gel stained with Coomassie brilliant blue for expressed proteins during expression and purification of rAsp fl 1 . Lane M, molecular mass markers; lane 1, non-I PTG-induced; lane 2, IPTG-induced rAsp fl 1; lane 3, rAsp fl 1 purified after affinity chromatography. (B) Immunodetection of rAsp fl 1. M, molecular mass markers; lane 1, Coomassie blue-stained rAsp fl 1; lane 2, rAsp fl 1 immunoblotted with serum pool from allergic patients.

that it may show allergenic cross-reactivity with Pen c 1 (see below). However, Asp fl 1 showed a much lower degree of sequence identity with subtilisin which by itself was a well characterized occupational allergen in the detergent industry (27).

\section{Expression and Purification of rAsp fl 1}

The Asp fl 1 cDNA fragment was synthesized by using PCR amplification of the Asp fl 1 open reading frame of the plasmid. The PCR product was purified, double-digested with $\mathrm{BamHI}$ and Hind III and then ligated into BamHI/HindlII sites of the plasmid pQE-30 coding for a His-tag fusion protein and expressed in a bacterial system. Optimal expression was obtained after a two-hour induction by IPTG (Fig. 4A). It can be clearly seen that the induced protein with an apparent molecular mass of $36 \mathrm{kDa}$ is prominent among expressed proteins. Purification of Asp fl 1 fusion protein was further carried out by metal chelate affinity chromatography. To investigate whether IgE antibodies were elicited in response to the Asp fl 1 in patients, the immunoblot in Fig. 4B was performed using serum I gE from a pool sera of patients with mold hypersensitivity to determine the specificity of IgE binding. The purified rAsp fl 1 showed strong reaction to sera IgE from patients with allergy (Fig. 4B), indicating the antigenic properties are well preserved in the recombinant derived protein.

\section{I mmunoblot-I nhibition Assays}

Preincubation of Asp fl 1-sensitive patients' sera pools with purified rAsp fl $1(10 \mu \mathrm{g} / \mathrm{ml})$ completely inhibited IgE binding to the natural Asp fl 1, whereas no reduction in intensity of the allergen band was observed after incubation with BSA and the buffer control (Fig. 5A). The result indicated that the recombinant derived Asp fl 1 contains all the IgE epitopes recognized by the sera from patients allergic to Aspergillus when comparing with its natural counterpart. Similar experiments with sera pools from patients allergic to Penicillium citrinum showed a strong depletion of IgE-binding Pen Cl 33 kDa band, when sera were preincubated with rAsp fl 1 (Fig. 5B). Therefore judging from inhibition experiments with rAsp fl 1, it is possible that common IgE epitopes may exist between allergens of $A$. flavus and $P$. citrinum.

\section{Sequence Comparison and Phylogenetic Relationships among Different Serine Proteases}

A search in the sequence database indicated that the deduced amino acid sequence of the serine protease from $A$. flavus exhibited a high degree of sequence similarity to previously cloned serine proteases. As shown in Fig. 6, the Asp fl 1 and serine proteases from $A$. oryzae and $A$. fumigatus form an independent and separate branch in the phylogenetic tree, pointing to the fact that the Asp fl 1 from A. flavus is apparently more closely related to the $A$. oryzae and $A$. fumigatus than to most other serine proteases.

\section{CONCLUSION}

We present here our successful attempt to characterize the allergens of Asp fl 1 by CDNA cloning, sequencing and expression in E. coli. This finding demonstrates that the product of rAsp fl 1 gene has a high potency of reactivity with IgE. This approach affords a facile process for the production of well-defined homogenous allergens. Recombinant allergens with high purity and quantity may therefore be very useful for a detailed study of epitope structure as well as the de-

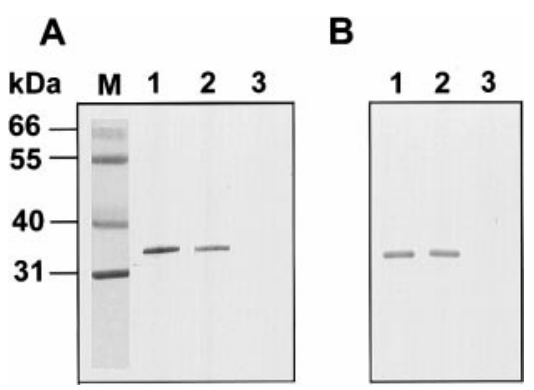

FIG. 5. Specific inhibition of IgE immuno-reactivity to native Asp fl 1 and Pen c 1 by recombinant allergen (rAsp fl 1). Preincubation of pooled sera with $r$ Asp fl 1 was performed as described in the Materials and Methods section. (A) native Asp fl 1, and (B) native Pen $c 1$ was used for SDS-PAGE and then electroblotted onto PVDF membrane. Lane $\mathrm{M}$, molecular mass markers; lane 1, preincubation of pooled sera with PBS buffer control in the absence of rAsp fl 1; lane 2 , preincubation of pooled sera with BSA in the absence of rAsp fl 1; lane 3, preincubation of pooled sera with rAsp fl 1 . 


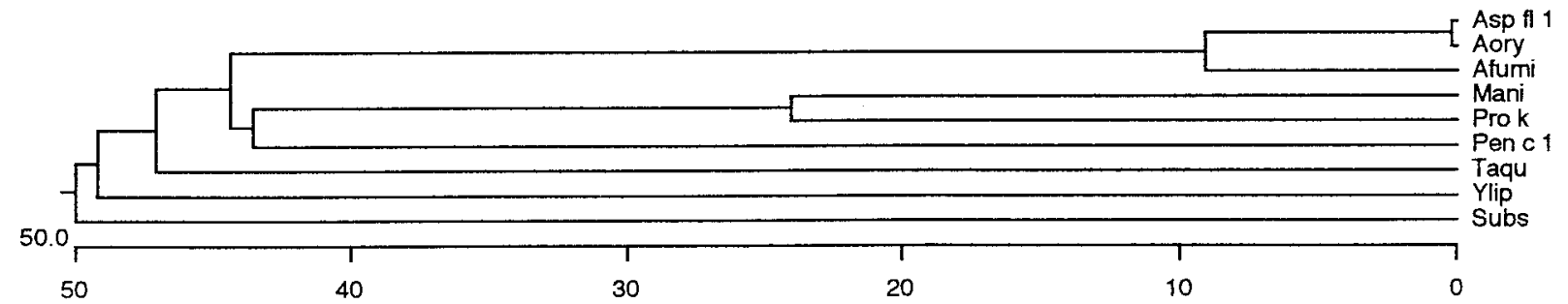

FIG. 6. Construction of a phylogenetic tree from amino acid sequences of different serine proteases. The tree was constructed based on the percentage divergence between protein sequences using a combination of distance matrix and approximate parsimony methods according to the phylogeny generation program of Hein (1990) (17). This program carries out multiple alignment by imposing restrictions based on the evolutionary relatedness of the aligned sequences. The length of each pair of branches represents the estimated sequence distance between aligned pairs. The origins of sequences and their accession numbers are as follows: Aory (accession No. P12547), Afumi (accession No. P28296), Mani (accession No. P29138), Prok (accession No. P06873), Pen c 1 (accession No. AF 084546), Taqu (accession No. CAA30559), Ylip (accession No. P09230), and Subs (accession No. P00782).

velopment of accurate diagnosis protocols leading to effective therapeutic strategies.

\section{REFERENCES}

1. Burge, H. A. (1985) Clin. Rev. Allergy 3, 319-329.

2. Bennett, J. E. (1990) in Principles and Practice of Infectious Disease (Mandell, G. L., Douglas, R. G., and Bennett, J . E., Eds.), pp. 1958-1961, Churchill Living-Stone, New York.

3. Arruda, L. K., Platts-Mills, T. A., Fox, J. W., and Chapman, M. D. (1990) J . Exp. Med. 172, 1529-1532.

4. Arruda, L. K., Mann, B. J ., and Chapman, M. D. (1992) J . Immunol. 149, 3354-3359.

5. Kumar, A., Reddy, L. V., Sochanik, A., and Kurup, V. P. (1993) J . Allergy Clin. I mmunol. 91, 1024-1030.

6. Banerjee, B., Kurup, V. P., Phadnis, S., Greenberger, P. A., and Fink, J . N. (1996) J . Lab. Clin. Med. 127, 253-262.

7. Lopez-Medrano, R., Ovejero, M. C., Calera, J . A., Puente, P., and Leal, F. (1995) I nfect. I mmun. 63, 4774- 4780.

8. Holdom, M. D., Hay, R. J ., and Hamilton, A. J . (1995) Free Rad. Res. 22, 519-531.

9. Horner, W. E., Helbling, A., Salvaggio, J. E., and Lehrer, S. B. (1995) Clin. Microbiol. Rev. 8, 161-179.

10. Su, N. Y., Yu, C. J., Shen, H. D., Pan, F. M., and Chow, L. P. (1999) Eur. J . Biochem. 261, 115-123.

11. King, T. P., Hoffman, D., Lowenstein, H., Marsh, D. G., PlattsMills, T. A. E., and Thomas, W. (1994) Int. Arch. Allergy I mmunol. 105, 224-233.

12. Chow, L. P., Fukaya, N., Miyatake, N., Horimoto, K., Sugiura, Y., Tabuchi, K., Ueno, Y., and Tsugita, A. (1995) J . Biomed. Sci. 2, 343-352.
13. Laemmli, U. K. (1970) Nature 227, 680- 685.

14. Tatsumi, H., Ogawa, Y., Murakami, S., Ishida, Y., Murakami, K., Masaki, A., Kawabe, H., Arimura, H., Nakano, E., and Motai, H. (1989) Mol. Gen. Genet. 219, 33-38.

15. Tinghino, R., Barletta, B., Palumbo, S., Afferni, C., I acovacci, P., Mari, A., Di Felice, G., and Pini, C. (1998) J. Allergy Clin. Immunol. 101, 772-777.

16. Vallverdu, A., Asturias, J . A., Arilla, C., Gomez-Bayon, N., Martinez, A., Martinez, J ., and Palacios, R. (1998) J. Allergy Clin. Immunol. 101, 363-370.

17. Hein, J . (1990) Methods Enzymol. 183, 626- 645.

18. von Heijne, G. (1986) Nucleic Acids Res. 14, 4683- 4690.

19. Hattori, T., Ichihara, S., and Nakamura, K. (1987) Eur. J . Biochem. 166, 533-538.

20. Ikemura, H., Takagi, H., and Inouye, M. (1987) J . Biol. Chem. 262, 7859-7864.

21. Shinde, U., and I nouye, M. (1994) J . Biochem. 115, 629- 636.

22. J aton-Ogay, K., Suter, M., Crameri, R., Falchetto, R., Fatih, A., and Monod, M. (1992) FEMS Microbiol. Lett. 92, 163-168.

23. Wells, J . A., Ferrari, E., Henner, D. J ., Estell, D. A., and Chen, E. Y. (1983) Nucleic Acids Res. 11, 7911- 7925.

24. Estell, D., Graycar, T. P., Miller, J . V., Powers, D. B., Burnier, J. P., and Wells, J. A. (1986) Science 233, 659- 663.

25. Kolattukudy, P. E., Lee, J. D., Rogers, L. M., Zimmerman, P., Ceselski, S., Fox, B., Stein, B., and Copelan, E. A. (1993) I nfect. I mmun. 61, 2357-2368.

26. Robinson, B. W., Venaille, T. J ., Mendis, A. H., and McAleer, R. (1990) J . Allergy Clin. Immunol. 86, 726-731.

27. Belin, L. G., and Norman, P. S. (1977) Clin. Allergy 7, 55- 68. 